\title{
Lower oesophageal sphincter tone in patients with peptic stricture
}

\author{
R LOBELLO, ${ }^{1}$ M STEKELMAN, AND D A W EDWARDS ${ }^{2}$ \\ From the Surgical Unit, University College Hospital Medical School, the Department of \\ Gastroenterology, Central Middlesex Hospital, London, and the Institute of Surgical Anatomy, \\ 2nd Medical Faculty, University of Naples, Naples, Italy
}

Lobello, R, Stekelman, M, and Edwards, D A W (1978). Thorax, 33, 574-578. Lower oesophageal sphincter tone in patients with peptic stricture. Radiographic studies of 161 patients with a stricture within the cardiac sphincter segment showed that in appropriate circumstances all patients could herniate stomach and reflux barium freely, but that the sphincter could obliterate the lumen and sustain this contraction by its basal tone in at least 122 . The sphincter could also relax and contract in response to a swallow. The capacity to remain closed depended on the position of the patient and on circumstances in which hiatal flow and reflux did not occur. Manometric studies of the cardiac sphincter were made in a further 14 patients with a stricture in the sphincter segment, and in appropriate circumstances the "basal tone" of the sphincter was found to be within our range of normal.

The lower oesophageal sphincter is claimed to be the chief factor in the control of reflux, and reflux oesophagitis is presumed to be a consequence of low sphincter tone. Many manometric studies of patients with reflux have deliberately excluded patients with stricture, but our radiological observations of such patients indicate that the sphincter can contract and relax. The purpose of this investigation was to quantify this contraction and relaxation in a group of patients who had a stricture within the sphincter segment.

\section{Methods}

\section{RADIOGRAPHIC}

The patient lies $10^{\circ}$ head down supine and drinks barium continuously (Edwards, 1969). The oesophagus relaxes proximal and distal to the stricture and is well distended, outlining the stricture as a section of increased resistance to stretch. The patient is then instructed not to swallow except on command. A peristaltic wave is followed down the oesophagus; the sphincter segment is identified immediately proximal to the herniated loculus of stomach and is seen to contract as the peristaltic wave runs into it. The oesophageal wall proximal

${ }^{1}$ Professor Lobello was in receipt of a European Science Exchange Program Fellowship.

${ }^{2}$ Work undertaken on behalf of the Medical Research Council. to the sphincter then relaxes, but the sphincter $\stackrel{\circ}{\stackrel{\varnothing}{\varnothing}}$ may or may not remain closed, depending on the $\overrightarrow{\vec{A}}$ pressure in the herniated stomach (Edwards, 1961). $\frac{3}{3}$ In these circumstances the mean length of the sphincter, by comparison with a marker in the oesophagus and correction for image magnification, is about $18 \mathrm{~mm}$. By comparison the length of a stricture is about $5 \mathrm{~mm}$. The site of the stricture $\underset{x}{\stackrel{0}{x}}$ in relation to the sphincter can be seen when theo wall of the sphincter relaxes in response to a swallow and stretches to the same bore as theô oesophagus, whereas the strictured section fails to stretch to the same degree. Fluoroscopy was re-음 corded on video tape for repeated study of the $D$ dynamics of the sphincter and stricture. A typicalo sequence is illustrated in fig 1 .

\section{MANOMETRIC}

Because the pressure recorded from a side-hole ${ }_{W}^{N}$ recording tube tends to vary with the orientationo of the opening, and because multiple tube as semblies are thick enough to obstruct the lumen of some strictures we used a single radio-opaque, infused, end-hole tube, $1.5 \mathrm{~mm}$ OD $\times 1 \mathrm{~mm}$ bore. 7 The pressure developed at the single end holeo seems likely to approximate to the mean radia pressure, but may be rather less since the tubed is $1.5 \mathrm{~mm}$ diameter instead of the conventiona $4 \mathrm{~mm}$ diameter of a multi-tube assembly, and 


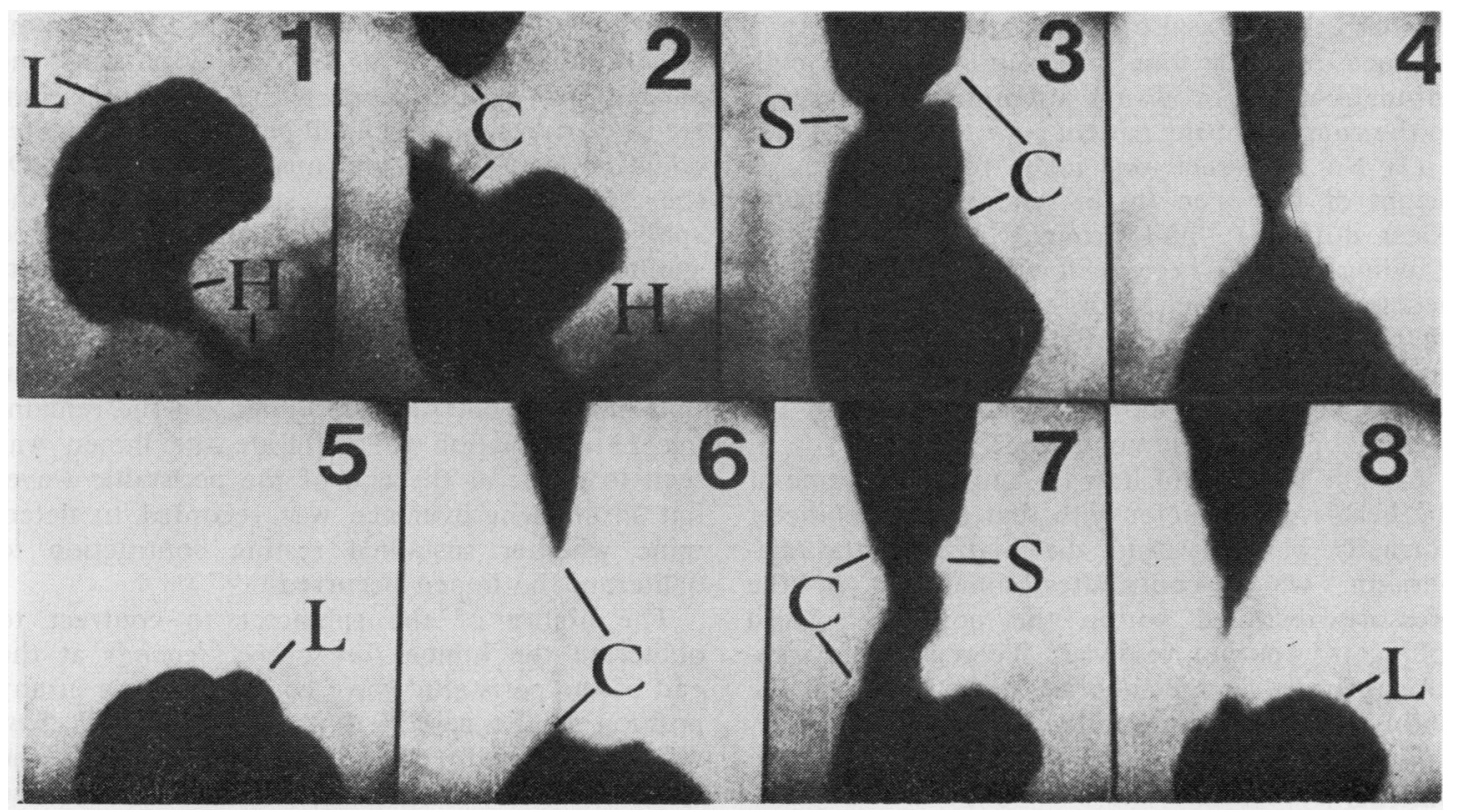

Fig 1 Sequence of cine frames to illustrate behaviour of sphincter containing a stricture. Patient supine, head down $10^{\circ}$. $S=$ stricture, $C=$ cardiac sphincter, $H=$ hiatus, $L=$ hiatal hernia. (1) Sphincter is closed, loculus full; (2) Patient has swallowed, sphincter relaxes and barium begins to flow back to meet barium pushed ahead by peristaltic wave; (3) Oesophagus and sphincter are same bore except for strictured segment; (4) Peristaltic wave empties oesophagus and sphincter; (5) Empty oesophagus and sphincter; (6) Another swallow pushes barium uphill; (7) Sphincter has relaxed and is being distended by barium but stricture can be seen; and (8) Sphincter is closed again.

there is a freer escape route for the infused fluid down the lumen of the sphincter.

The tube was passed down the oesophagus in the fasting subject and an attempt was made to pass it through the hiatus into the abdominal stomach. Success in reaching the abdominal stomach is unusual unless the herniated loculus is less than 1.5 to $2 \mathrm{~cm}$ in diameter. The tube was constantly infused at $0.4 \mathrm{ml} / \mathrm{min}$ by a syringe pump and withdrawn in $0.5 \mathrm{~cm}$ steps. Swallows were recorded at each step.

Some methodological problems were considered.

(1) If the hiatus is large when the patient is supine and there is adequate liquid or gaseous contents in the stomach to allow transmission of abdominal pressure through the hiatus to the intrathoracic part of the stomach (Edwards, 1973) the sphincter may be exposed to and challenged by the difference in pressure between abdomen and thorax. If the power of the contraction of the sphincter is inadequate to overcome this the sphincter lumen will be open, and pressure recorded from within it will be the same as that in the herniated stomach. This would give an apparent sphincter tone of zero. To record the sphincter tone the patient must be in a position in which there is minimum challenge to the sphincter or possibility of reflux. The tube was pulled up in $0.5 \mathrm{~cm}$ steps, and repeated records were therefore obtained with the patient sitting or standing, as well as lying down, so far as the condition, tolerance, agreement, and ready co-operation of the patient permitted.

(2) When the sphincter is herniated into the chest and surrounded by a negative pressure how should its power of squeeze be expressed? Is the conventional reference to subhiatal fundic pressure appropriate, or should the squeeze be measured against the ambient intrathoracic pressure, or should the difference in pressure between contraction and relaxation be recorded? Pressure was measured as "endexpiratory," and in each record the height of the sphincter pressure was referred to $(a)$ the fundal pressure or loculus pressure (EE-Abd), $(b)$ the pressure recorded from the body of the oesophagus (EE-Oes), or (c) the amount of relaxation recorded (EE-Relax). The "pressure" recorded at the level of the hiatus was referred to the fundal pressure. Since the purpose of the study was to 
establish that a basal tone was present rather than to measure this tone precisely, repeated pullthroughs were not always attempted in deference to the comfort of the patient.

(3) No allowance was made for the changing height of the open tip in relation to the transducer during a "pull-through" in the sitting or standing position because it was found to be unnecessary over such short distances. The oesophagus and stomach can be likened to a column of water, and when the tip of the manometry tube was moved up and down in such a column the recorded pressure did not change.

(4) The problem of how to compare the tone of the herniated sphincter with that of the sphincter normally placed within the hiatus of the diaphragm, which contributes something to the pressure recorded within the normally placed sphincter, was not resolved. We report the pressure recorded as the tube was pulled through the hiatus and the pressure in the sphincter separately.

\section{Patients}

\section{RADIOGRAPHIC STUDIES}

The records of screening 260 patients with peptic stricture by the same observer (DAWE) were reviewed for information on sphincter behaviour. None of these patients had systemic sclerosis or surgical damage to the cardiac sphincter or hiatus at the time of the examination. All had had an endoscopy that confirmed ulcerative oesophagitis and obstruction to the instrument.

\section{MANOMETRIC STUDIES}

Sphincter pressure was measured in 14 consecutive patients seen in the clinic who satisfied the following criteria:

(1) They had radiographic evidence of a peptic stricture within the sphincter segment at the time of the manometric study.

(2) There was endoscopic evidence of oesophagitis and obstruction to the oesophagoscope just before the manometric study.

(3) They were willing to co-operate in the study and able to swallow the manometry tube without distress.

(4) We were able to pass the manometry tube through the stricture into the loculus.

\section{Results}

RADIOGRAPHIC STUDIES

Of the 260 patients not subjected to manometry, the stricture was identified as in the sphincter seg- ment in 161 , above the sphincter in 52 , and mar- $\frac{\text { की }}{\frac{0}{0}}$ ginally in or above in 47. In all patients the capacity to herniate stomach and to reflux barium $\frac{\bar{\sigma}}{2}$ freely was present, and in all patients the sphincter $\widehat{D}$ could be identified by its capacity to contract. Of $\curvearrowright$ the 161 patients with a stricture within the ${ }^{\circ}$ sphincter segment, 122 had evidence of sustained $\overrightarrow{0}$ sphincter contraction at rest sufficient to obliter- $\overrightarrow{\vec{\omega}}$ ate the lumen, including the lumen of the stricture, $\stackrel{\omega}{\sigma}$ together with an ability to relax in response to a $\overrightarrow{\vec{\gamma}}$ swallow. In six there was evidence of contraction $\underset{\omega}{x}$ that did not obliterate the lumen. In the remain- $\omega$ ing 33 contraction to obliterate the lumen was or seen to occur at the end of the peristaltic wave, $\rightarrow$ but insufficient evidence was recorded to deter-o mine whether sustained resting contraction to obliterate the lumen occurred.

The ability of the sphincter to contract to obliterate the lumen for several seconds at the end of the peristaltic wave could be shown in any ㄴ. position of the patient if it occurred at all. Sus- $\vec{\varphi}$ tained contraction, caused by the basal tone of the 0 o sphincter, could often only be recorded with the patient in a particular position, or in circumstances in which hiatal flow and reflux did not occur (Edwards, 1969). These radiographic studies suggested $(a)$ that the presence of active oesophagitis $\frac{\circ}{\Phi}$ and healing with fibrosis did not destroy the ability of the sphincter segment to maintain a basal tone 0 and to relax and contract in response to a swallow, $\frac{3}{3}$ and $(b)$ that sustained closure of the sphincter was related to the absence of hiatal flow of barium.

The radiographic behaviour of the sphincters of the additional 14 patients subjected to manometry was similar.

\section{MANOMETRIC STUDIES}

Fourteen patients were examined sitting or stand- $\frac{\circ}{3}$ ing; in nine it was possible to pass a tube through the hiatus and evidence of a hiatal high pressure zone was obtained; seven were also examined supine. All showed evidence of a sustained sphincteric high pressure zone with relaxation and $N$ "after swallow contraction" in response to a N swallow (see fig 2).

The results are shown graphically in fig 3. The mean of the recorded sphincter pressures, when the abdominal pressure was used as a base line,, was $1.05 \mathrm{kPa}$ standing and 1.25 supine; when the oesophageal pressure was the base line, 1.26 stand -ing and 1.48 supine; when relaxation pressure was $\frac{0}{\circ}$ substracted from contraction pressure, 0.8 standing and 1.13 supine; and the mean hiatal pressure $\frac{?}{\mathbb{P}}$ was 0.64 standing and $0.5 \mathrm{kPa}$ supine. Using the paired value " $t$ " test there was no difference between these various values at the $5 \%$ level of sig- $\bigcirc$ 
$1 \mathrm{kPa} \ 1$<smiles>CC(C)C</smiles>

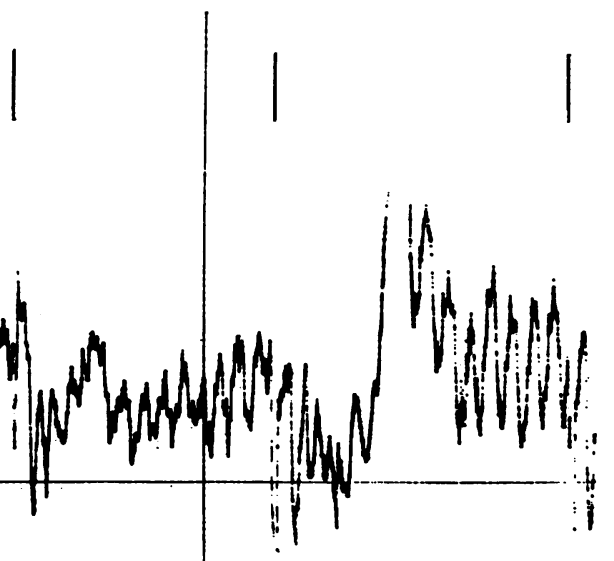

Fig 2 Stepwise pull-through record to illustrate basal sphincter tone, relaxation, and "after swallow contraction" in response to a swallow in patient with a stricture in sphincter segment. Pressure calibration $1 \mathrm{kPa}$. Long vertical line indicates when tube was withdrawn $0.5 \mathrm{~cm}$; a short line indicates a swallow.

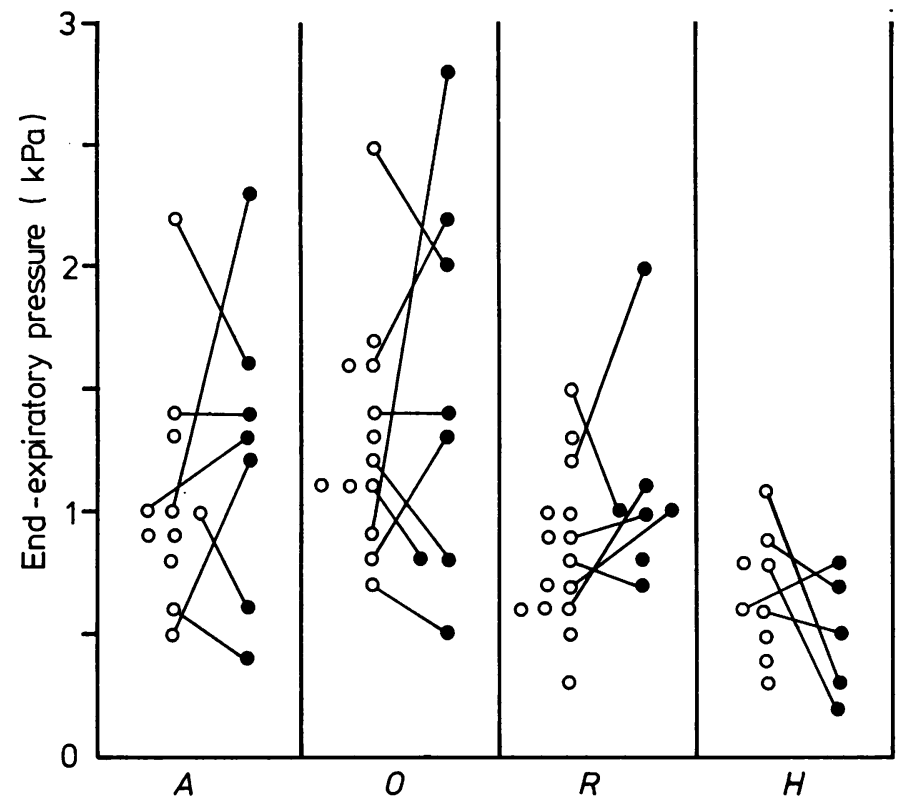

Fig 3 End-expiratory pressures in $\mathrm{kPa}$ of 14 patients with a stricture within sphincter segment (mean, or single value if only one obtained). $\mathrm{O}=$ pressure when patient is standing or sitting, $\bigcirc=$ pressure when same patient is supine. Column A, sphincter pressure referred to fundal pressure; column $O$, sphincter pressure referred to mid-oesophageal pressure; column $R$, difference between sphincter contraction and relaxation pressures; column $H$, pressure recorded from walls of hiatal canal referred to fundal pressure.

nificance. Detailed information is given in the table.

In a previous study (Dilawari et al, 1974) a group of 163 patients without radiographic reflux or oesophagitis had the distribution of sphincter pressure seen in open columns in fig 4. These pressures were recorded with an assembly of three or four infused tubes with side holes, and the value plotted was the mean of the maximum endexpiratory pressure recorded in each of the three or four channels, referred to fundic pressure. The mean value for each of our 14 patients is shown as a black square. The distribution of pressures of strictured sphincters, which are isolated from the effect of the hiatus, is similar to that of the patients without radiographic reflux. 
Table Pressure recordings in 14 subjects

\begin{tabular}{|c|c|c|c|c|}
\hline Patient & $E E$ abd & EE oes & EE relax & Hiatal \\
\hline 1 & $8, \bar{x} 8(23, \bar{x} 15)$ & $9, \bar{x} 8(28, \bar{x} 19)$ & $7, \bar{x} 7(10, \bar{x} 9)$ & $6, \bar{x} 6(8, \bar{x} 8)$ \\
\hline 2 & $5, \bar{x} 5(12, \bar{x} 8)$ & $7, \bar{x} 7(13, \bar{x} 12)$ & $6, \bar{x} 6(11, \bar{x} 10)$ & $10, \bar{x} 9(10, \bar{x} 7)$ \\
\hline 3 & $10, \bar{x} 7(6, \bar{x} 6)$ & $11, \bar{x} 11(8, \bar{x} 8)$ & $9, \bar{x} 9(-)$ & $14, \bar{x} 11(3, \bar{x} 3)$ \\
\hline 4 & $10, \bar{x} 10(13, \bar{x} 11)$ & $16, \bar{x} 15(22, \bar{x} 22)$ & $12, \bar{x} 11(20, \bar{x} 20)$ & $6, \bar{x} 6(5, \bar{x} 5)$ \\
\hline 5 & $22(16)$ & $25(20)$ & $15(10)$ & - \\
\hline 6 & $14(14)$ & $14(14)$ & $9(10)$ & - \\
\hline 7 & $6, \bar{x} 6(4)$ & $7, \bar{x} 6(5)$ & $8, \bar{x} 7(7)$ & $9, \bar{x} 8(2)$ \\
\hline 8 & $13, \bar{x} 12$ & $16, \bar{x} 13$ & $10, \bar{x} 10$ & 5 \\
\hline 9 & - & 8 & 3 & - \\
\hline 10 & 9 & $11, \bar{x} 10$ & $5, \bar{x} 5$ & 3 \\
\hline 11 & 9 & $17, \bar{x} 13$ & 13 & $7, \bar{x} 4$ \\
\hline 12 & - & - & 7 & - \\
\hline 13 & $10, \bar{x} 9$ & $12, \bar{x} 10$ & $6, \bar{x} 6$ & 8 \\
\hline 14 & - & 13 & 10 & 一 \\
\hline
\end{tabular}

$\mathrm{EE}$ abd = End-expiratory pressure in sphincter compared with the fundal (abdominal) pressure.

EE oes=End-expiratory pressure in sphincter compared with the pressure in the body of the oesophagus.

EE relax = End-expiratory pressure during relaxation subtracted from end-expiratory pressure during resting contraction.

Hiatal =End-expiratory pressure in the hiatus compared with fundal pressure.

$\overline{\mathbf{x}}=$ Mean.

Values outside brackets were obtained with the patient sitting up, those within the brackets with the patient lying down. The first figure is the maximum pressure recorded, that following $\overline{\mathbf{x}}$ is the mean if more than one measurement was obtained.

All pressures in $\mathbf{k P a} \times 10^{-1}$.

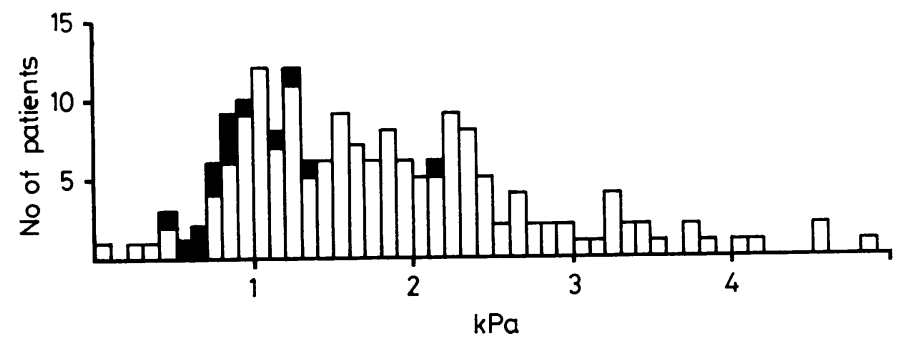

Fig 4 Sphincter pressures of patients without radiographic reflux (open columns) and of the 14 patients with stricture (solid squares).

\section{Conclusions}

These studies suggest:

(1) that the pressure recorded when the subject is sitting or standing is not materially different from that when the subject is supine;

(2) that by using oesophageal pressure as a reference a slightly higher sphincter pressure might be recorded, but that the difference is not significant with the small numbers measured;

(3) that the walls of the hiatal canal produce a squeeze or resistance to stretch comparable with, though possibly less than, the resistance to stretch recorded from the herniated sphincter separated from its hiatus (cf Habibulla, 1972);

(4) that the distribution of pressures recorded from the herniated sphincter, separated from ambient hiatal squeeze and containing a stricture within it, is similar to the distribution of sphincter pressures of patients without oesophagitis or radiographic reflux; and

(5) that sphincter tone is not a prime factor in the development of reflux leading to oesophagitis and peptic stricture formation.

\section{References}

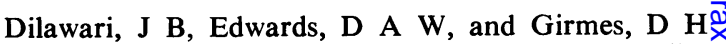
(1974). The probability of symptoms or of radio-o graphic evidence of reflux predicted by lower 3 . oesophageal sphincter pressure. Proceedings of the Fourth International Symposium on Gastrointestinal Motility, edited by E E Daniel, pp 441-448. Mitchell Press, Vancouver.

Edwards, D A W (1961). The anti-reflux mechanisms:D manometric and radiological studies. British Journale of Radiology, 34, 474-487.

Edwards, D A W (1969). Text Book of X-ray Diagnosis, of edited by $S C$ Shanks and $P$ Kerley, 4th edition, $N$ pp 266-319. H K Lewis and Co, London.

Edwards, D A W (1973). Symposium on gastro- esophageal reflux and its complications. Gut, 14, 233-237.

Habibulla, K S (1972). The diaphragm as an antireflux barrier. Thorax, 27, 692-702.

Requests for reprints to: D A W Edwards, Surgica $\mathbb{B}$ Unit, University College Hospital Medical Schoolo University Street, London WC1E 6JJ. 